\title{
Private Beteiligung an der Griechenlandrettung: Erfreuliche Mogelpackung!
}

Am 21. Juli haben die Regierungschefs der Euroländer auf ihrem Gipfeltreffen zusammen mit der EU-Kommission, der Europäischen Zentralbank (EZB) und dem Internationalen Währungsfonds (IWF) ein zweites Rettungspaket für Griechenland (109 Mrd. €) beschlossen. Im Gegensatz zum ersten Rettungspaket wurde dabei richtigerweise auf niedrige Zinssätze und längere Laufzeiten der Rettungskredite geachtet. Zudem soll die Europäische Finanzstabilisierungsfazilität (EFSF) Anleihen direkt am Sekundärmarkt ankaufen können.

Auch die deutsche Forderung nach einer stärkeren Beteiligung privater Gläubiger - vor allem Banken und Versicherungen - wurde berücksichtigt: Diese sollen sich „freiwillig“ an der Stützung beteiligen. Dazu hat das Institute of International Finance (IIF) vier Beteiligungsvarianten ausgearbeitet, bei denen sich rechnerisch jeweils ein Barwertverlust von $21 \%$ ergibt. Würden sich private Gläubiger zu $90 \%$ beteiligen und dabei alle vier Varianten gleichmäßig nutzen, würde sich die Schuldenlast Griechenlands effektiv um 13,5 Mrd. € verringern.

Kern aller vier Beteiligungsvarianten ist der Umtausch aktueller Griechenlandanleihen gegen solche mit längerer Laufzeit und festgelegten Zinssätzen („Kupon“). Bei Variante 1 tauscht der Gläubiger seine Anleihen sofort gegen solche mit einer Laufzeit von 30 Jahren und einem Durchschnittszins von 4,5\%. Variante 2 unterscheidet sich von der ersten nur darin, dass der Umtausch erst bei Fälligkeit der alten Anleihen erfolgt. Bei beiden Varianten erfolgt der Umtausch zum Nominalwert: Wurde die Anleihe zu $100 €$ ausgegeben, bekommt der Gläubiger eine neue Anleihe in Höhe dieses Wertes, auch wenn der derzeitige Kurswert niedriger ist. Damit bleibt die nominale Schuldenlast Griechenlands erhalten.

Lediglich bei den weiteren Varianten verringert sich die griechische Schuldenlast, da dort der (sofortige) Umtausch mit einem Abschlag von $20 \%$ auf den Nominalwert erfolgt: Für eine Anleihe in Höhe von nominal $100 €$ erhält der Gläubiger nur eine in Höhe von $80 €$. Um den Wertverlust auszugleichen, sieht Variante 3 einen höheren Zins von 6,42 \% und Variante 4 einen Zins von 5,9 \% bei einer Laufzeit von nur 15 Jahren vor.

Attraktiv ist die Beteiligung für die privaten Gläubiger in allen Fällen: Sie können Anleihen, die derzeit ein Rating auf Ramschniveau haben, gegen solche mit erstklassigem AAA-Rating tauschen. Denn die neuen Staatsanleihen sind in Variante 1 bis 3 alle vollständig (durch Zerobonds des EFSF) und in Variante 4 immerhin teilweise abgesichert. Lediglich für die Zinszahlungen besteht dann noch ein Ausfallrisiko.

Der „Verlust“ der privaten Gläubiger von jeweils $21 \%$ ergibt sich - neben dem Abschlag in den letztgenannten Varianten - durch die Verlängerung der Laufzeit: Da eine Anleihe mit späterer Rückzahlung heute weniger wert ist, sinkt deren Barwert - und zwar auf genau $79 \%$, wenn man die Annahmen des IIF akzeptiert (Abzinsfaktor von $9 \%$ auf die Zinszahlungen).

Das heißt aber nicht, dass es bei privaten Gläubigern notwendigerweise zu einem Bilanzverlust in dieser Höhe kommt - ganz im Gegenteil! Abschreibungen muss ein Finanzinstitut nur vornehmen, wenn die alten Anleihen aktuell mit mehr als $79 €$ (für nominal 100) bilanziert sind. Institute, die nach IFRS („At Fair Value“) bilanzieren oder ihre Griechenlandanleihen im Handelsbuch oder der Liquiditätsreserve nach Vorschriften des Handelsgesetzbuches (HGB) führen, müssen diese ohnehin schon auf den Kurswert abgeschrieben haben. Aktuell (August 2011) liegen die Kurswerte griechischer Anleihen mit einer Fälligkeit zwischen 2012 und 2020 zwischen 47 und $71 €$. Beim Umtausch sind also massive Bilanzgewinne zu erwarten!

Hilft es Griechenland? Eine effektive Senkung der Schuldenlast für Griechenland findet nur bei den Varianten 3 und 4 statt, bei denen die Gläubiger auf $20 \%$ der Nominalschuld verzichten. Doch weshalb sollte ein privater Investor diese Varianten wählen? Die höheren Zinszahlungen dieser Varianten unterliegen einem Ausfallrisiko, und bei Variante 4 ist die Rückzahlung nur teilgarantiert. Die meisten Investoren dürften sich daher für Variante 1 entscheiden. Dort ist die Rückzahlung sofort mit dem Umtausch über den EFSF gesichert. Die versprochenen 13,5 Mrd. € Entlastung für Griechenland sind damit unrealistisch.

Fazit: Griechenland wird durch die Beteiligung privater Gläubiger kaum entlastet und den Banken winken hohe Bilanzgewinne. Ist das Ganze also eine Mogelpackung? Leider ja - trotzdem ist sie zu begrüßen! Denn erstens hat die Mogelpackung der deutschen Regierung geholfen, dem erneuten Rettungspaket für Griechenland ohne Gesichtsverlust zuzustimmen. Sollte zweitens die vom IIF erwartete hohe Beteiligung des Privatsektors erreicht werden, würde sich zudem auch das Problem verringern, dass Zahlungsprobleme Griechenlands direkt das Bankensystem des gesamten Euroraumes gefährden.

Befriedigend ist die Lösung aber nicht. Wie alle bisherigen Rettungsaktionen leidet auch das neue Rettungspaket unter nationalen Querschüssen und halbherziger Durchführung, die zu wenig effektiven und unnötig teuren Kompromissen geführt haben. Daran dürfte sich künftig auch wenig ändern. Eine Überwindung der Eurokrise bleibt damit gefährdet. Die Gipfelbeschlüsse tragen aber immerhin dazu bei, Zeit für grundlegende Reformen im Euroraum zu gewinnen - mit hoffentlich reduzierter Gefahr einer erneuten Finanzkrise.

Heike Joebges, Prof. Dr., lehrt Volkswirtschaftslehre mit dem Schwerpunkt International Economics an der Hochschule für Technik und Wirtschaft in Berlin. Arbeitsschwerpunkte: Finanz- und Währungskrisen, Handelsungleichgewichte, Exportorientierung Deutschlands. e-mail: Heike.Joebges@HTW-Berlin.de 